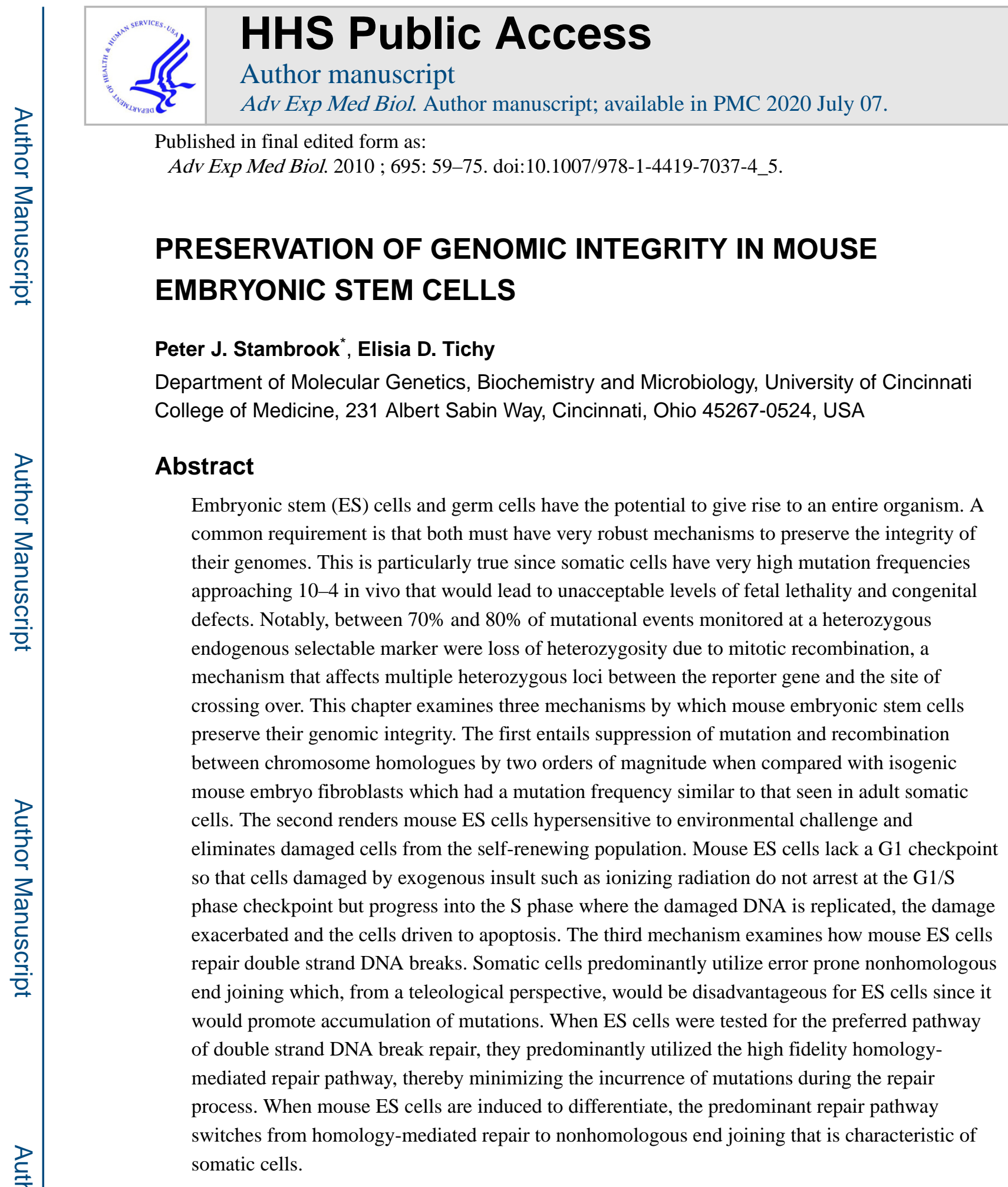

\title{
INTRODUCTION AND HISTORICAL PERSPECTIVE
}

The capacity of a single cell to give rise to multiple different cell types of an organism has been area of investigational interest for many years. A question that lingers is that of nuclear equivalence, (i.e.,whether all cells of a multicellular organism have genomes that are

\footnotetext{
"Corresponding Author: Peter J. Stambrook-Department of Molecular Genetics, Biochemistry and Microbiology, University of Cincinnati College of Medicine, 231 Albert Sabin Way, Cincinnati, Ohio 45267-0524, USA. stambrpj@ucmail.uc.edu.
} 
quantitatively and qualitatively equivalent). Nuclear equivalence was tested as early as 1902 when Spemann separated the two blastomeres following the first cleavage division of a newt embryo and showed that both blastomeres could develop into a complete embryo, supportive of functional equivalence (cited in ref. 1). This work was presaged eleven years earlier by Hans Driesch who showed that separated blastomeres of sea urchin embryos were capable of developing into normal, albeit smaller, sea urchins (cited in ref. 1).

Half a century later, a period replete with many important and insightful discoveries, two seminal experiments were described. The classic and elegant experiments by Hämmerling, using the green alga Acetabularium as a model organism, showed that the nucleus contained all of the information necessary to dictate cell morphology. ${ }^{2}$ Acetabularium is a single cell organism that has a base that contains the nucleus, a stalk and a cap. Using two species of Acetabularium, A. mediterrania and A. crenulata, the former with a smooth cap and the latter with a wrinkled cap, Hämmerling showed that when the stalk and cap of $A$. mediterrania was grafted to the base of $A$. crenulata, the grafted cap was transformed from smooth to wrinkled. The reciprocal experiment also held true, indicating that the genetic information determining cap morphology was dictated by the nucleus within the grafted base. At about the same time, Briggs and King expanded on the findings of Driesch and Spemann by successfully transferring the nucleus of an undifferentiated frog blastula cell to an enucleated fertilized egg. In about one third of their attempts, the transplanted nucleus was capable of directing development to a normal embryo. When using nuclei from later staged embryos (neurula or tailbud), however, they found that none of the recipient eggs developed normally and that the majority failed to complete gastrulation, ${ }^{4}$ suggesting a restriction in the potential to differentiate as cells mature.

In 1962, the question of nuclear equivalence and the potential pluripotency of nuclei from differentiated cells in a multicellular organism was reignited by the findings of John Gurdon. He reported that the nucleus from a Xenopus tadpole intestinal cell, when introduced into an enucleated Xenopus egg, was able to support the development of a fully-formed feeding tadpole, demonstrating that the intestinal cell nucleus retained the genetic information necessary to produce all of the cells of a complete multicellular organism. ${ }^{5}$ It should be noted, however, that of the large number of nuclear transplant experiments performed, only slightly more than one percent of recipient eggs successfully produced a mature tadpole, leading to discussions as to whether the nuclei with demonstrated pluripotency are derived from stem cells or come from truly differentiated cells.

A major advance in cloning technology occurred in 1981 when Martin Evans in Cambridge and Gail Martin in San Francisco concomitantly and independently succeeded in culturing embryonic stem (ES) cells from mouse blastocysts. ${ }^{6,7}$ The pluripotency of ES cells coupled with the ability to target specific gene sequences to their cognate sites by targeted homologous recombination led to cloning of mice with very specifically and selectively inactivated or modified genes. Oliver Smithies and his colleagues first used targeted homologous recombination to correct a mutant Hprt gene in mouse ES cells ${ }^{8}$ and independently Capecchi and colleagues inactivated an Hprt gene in a similar manner. ${ }^{9}$ Three years later reports of genetically modified mice produced by introduction of targeted ES cells into the blastocoel of recipient blastocysts appeared. ${ }^{10-12}$ The fact that mouse ES cells 
were able to support the development of an intact organism and enter the germ line without any sign of other developmental or physiological problems suggested that the ES cells had retained full genomic competency.

A test for a similar degree of nuclear equivalence in mammalian somatic cells came eight years later with the development of somatic cell nuclear transfer (SCNT) which essentially mimicked the experiments described by Gurdon. ${ }^{5}$ Ian Wilmut and colleagues ${ }^{13}$ transplanted the nucleus derived from a sheep mammary gland cell into an enucleated egg that was activated and allowed to develop to the blastocyst stage in vitro, at which time it was implanted into the uterus of a surrogate mother. A viable lamb was born and designated Dolly. The success rate from this procedure, however, was very low with only three of 277 transplanted blastocysts producing live births and only one (Dolly) surviving to adulthood. Since the initial report, however, SCNT has been applied successfully in a range of mammals extending from mice to horses to ferrets (cited in ref. 14). SCNT has also been used to produce ES cells that then were used to correct a genetic defect in a mouse model, ${ }^{15}$ suggesting a potential means for avoiding the requirement and attendant ethical dilemma, for using ES cells derived from fertilized eggs. Despite the successes of SCNT, problems with the technology remain. The frequency with which transplanted eggs reach blastula stage is low and in models in which animals are allowed to develop to maturity, there are frequent developmental abnormalities. In the case of Dolly, she was euthanized at half the age of the normal lifespan of a sheep. She suffered from arthritis not normally seen in animals her age and had progressive lung disease that on autopsy proved to be an adenocarcinoma. Dolly also had short telomeres characteristic of somatic cells of older animals, suggesting that either her telomeres had shortened prematurely, promoting rapid aging, or that the adult nucleus from which she had been derived already had eroded telomeres.

More recently, two laboratories independently showed that adult somatic cells, first from from mice ${ }^{16}$ and then from humans ${ }^{17,18}$ could be induced to dedifferentiate and produce pluripotent stem cells (IPS cells) that mimic ES cells in morphology and competence to rediffentiate into many cell types following the concomitant introduction of cDNAs encoding four genes. The genes that initially seemed sufficient for IPS induction were Oct4, Sox2, Klf4 and c-Myc, but additional genes such as Nanog and other members of the Klf family appear to also be important in the induction of IPS cells. The important issue in the context of this discussion is that the data reaffirm that somatic cells retain all of the nuclear information to potentially direct differentiation into multiple different cell types. Challenges still exist to regulate epigenetic changes that must occur during the dedifferentiation process and concerns about the extent of nuclear equivalence in somatic cells remain and are similar to those raised for nuclei used for SCNT.

Somatic cells and mouse ES cells are intrinsically different in the extent to which they retain genomic integrity. Implicit in this contention is that somatic cells, which form the basis for the SCNT and IPS technologies, lack the same degree of nuclear equivalence as true ES cells derived from blastulae which may, in part, account for the low efficiency of these procedures. This proposition is consistent with the argument that somatic cells are more tolerant of deleterious mutations than germ cells and, by extrapolation, ES, cells and that somatic mutations accumulate as a function of age ultimately leading to somatic disease and 
death. ${ }^{19-22}$ From an evolutionary and selfish perspective, this aging process may be viewed as described by Weil and Radman, "We have apparently evolved to spend most of our "life capital' for the selfish objective of evolution: maintaining the species through immortality of the germline and disposing of the postreproductive soma. Although evolution may not 'care' about our post reproductive soma, we certainly do." 23 It follows that ES cells, which share the characteristic of pluripotency with germ cells, have robust mechanisms for preserving the integrity of their genomes that are different from or augment those present in somatic cells. This discussion focuses principally on mouse ES cells and describes some of the strategies observed in ES cells that promote genomic integrity.

\section{MUTATION FREQUENCIES IN SOMATIC CELLS}

Mammalian somatic cells display very high mutation frequencies in vivo, approaching $10^{-4} \cdot 21,22,24,25$ Furthermore, between $70 \%$ and $80 \%$ of mutation events at heterozygous loci involve loss of heterozygosity ( $\mathrm{LOH}$ ) which occurs as a consequence of recombination between homologous chromosomes (mitotic recombination) and which affects all heterozygous loci proximal to the cross-over site. ${ }^{24-27}$ Mutation frequencies involving point mutations small insertions or deletions have been established using transgenic mice usually harboring prokaryotic reporters such as $l a c I,{ }^{26} l a c Z,{ }^{28} g p t^{29}$ and the $c 11$ gene of $\lambda$ phage, ${ }^{30}$ among others. In rodent models, the endogenous genes predominantly used as reporters are the selectable genes encoding the purine salvage enzymes hypoxanthine guanine phosphoribosyltransferase (Hprt) ${ }^{27}$ and adenine phosphoribosyltransferase (Aprt). ${ }^{25,27}$ Of these models, the Aprt model is the most effective in identifying events involving mitotic recombination which accounts for the majority of mutations at heterozygous loci in mouse and man. The strategy underlying this mouse model has been to cross mice of one strain (e.g., 129/sv) homozygous for a targeted null Aprt allele with mice of a second strain (e.g., C57B1/6) with wildtype Aprt. The F1 mice are obligate heterozygotes at Aprt as well as at multiple flanking polymorphic markers characteristic of each strain. At the age of 8 to 10 weeks, the mice are sacrificed and skin fibroblasts, usually from the ear and splenic T-cells are placed into culture and immediately in medium containing either 5-fluoroadenine or \%, 6 diaminopurine (DAP), both of which are cytotoxic to cells with functional Aprt activity and which allow only cells lacking Aprt to grow in culture. Colonies deficient in Aprt activity arise in the selection medium and are picked and assessed first for LOH at Aprt by PCR analysis. If it is determined that there is $\mathrm{LOH}$ at $A p r t$, the flanking polymorphic markers are further analyzed and if necessary Aprt is PCR amplified and sequenced. In this manner, virtually all mutational pathways leading to Aprt deficiency can be identified. Between $70 \%$ and $80 \%$ of events involve $\mathrm{LOH}$ at Aprt and at linked flanking polymorphic markers to a point proximal to Aprt where retention of heterozygosity is maintained, indicative of mitotic recombination. Other events such as interstitial deletion, gene conversion, point mutation or epigenetic inactivation of the functional allele constitute between $20 \%$ and $30 \%$ of the remaining mutational events.

\section{PROTECTION OF THE MOUSE ES CELL GENOME}

While mutation in the germline is important for genetic diversity and the evolution of species, a mutation frequency or $\mathrm{LOH}$ in the order of $10^{-4}$, such as that found in somatic 
cells (Fig. 1), would result in large numbers of defective embryos and births of individuals with congenital malformations.

The question arises whether pluripotent stem cells are protected from acquiring and accumulating mutations. At least three mechanisms by which this may occur have been described in mouse ES cells. These include suppression of mutation and recombination between chromosome homologues, elevated apoptosis and preferential utilization of high fidelity homology-mediated repair of DNA double strand breaks (DSBs) rather than error prone nonhomologous end joining (NHEJ) that is predominant in somatic cells. While these mechanisms have been described for mouse ES cells, they are not necessarily the same as those found in true germ cells or even in human ES cells. They do, however, provide insight into the importance for pluripotent cells to maintain genomic stability.

\section{The Frequency of Mutation Is Suppressed in Mouse ES Cells}

As indicted above, somatic cells have high mutation frequencies that would be detrimental to the reproductive and developmental functions of germ cells and pluripotent stem cells. Using the Aprt mouse model, ES cells were prepared from F1 blastocysts derived from a cross between 129 strain mice homozygous for the targeted null Aprt allele and C3H mice with wildtype Aprt Isogenic mouse embryo fibroblasts (MEFs) were prepared from 14 day old embryos for comparative purposes. The capacity for ES cells and MEFs to form Aprt or Hprt deficient colonies in DAP or in 6-thioguanine (6-GT) respectively, showed that mutation frequency at Aprt in ES cells $\left(\sim 10^{-6}\right)$ was about 100-fold lower than in MEFs $\left(\sim 10^{-4}\right)$, which approximated the in vivo mutant frequency observed in adult somatic cells (Fig. 2). The mutation frequencies at Hprt showed a similar trend, albeit with lower frequencies in all cases. This difference is attributed in part to the fact that Aprt is autosomal and is able to undergo mitotic recombination whereas Hprt is X-linked and is monosomic in males and effectively monosomic in females due to X-inactivation. About $60 \%$ of the mutation events detected involved LOH, but unlike MEFs and adult somatic cells, LOH was not predominantly due to mitotic recombination, but rather to nondisjunction resulting in uniparental disomy. ${ }^{31}$ The Aprt gene is on mouse chromosome 8 and it may be that nondisjunction involving this chromosome is unusually high, skewing the data and interpretation. ${ }^{32}$ Discordant data regarding mitotic recombination frequencies in mouse ES cells have been reported in a model in which genes encoding two different fluorescent proteins were targeted to the ROSA26 locus in each of the two chromosome 6 homologues.

${ }^{33}$ Conversion from dichromatic to monochromatic fluorescence, indicative of loss of one of the alleles, occurred with a frequency of about $10^{-4}$. About half of the events appear to be due to mitotic recombination since there was retention of heterozygosity at a locus proximal to ROSA26. Most of the other clones with monochromatic fluorescence had lost heterozygosity at ROSA26 and also at the centromeric locus, indicative of nondisjunction and chromosome 6 uniparental disomy. The recombination frequency is about two orders of magnitude higher than that observed with the Aprt model and may be reconciled by arguing that different chromosomes support very different recombination frequencies in ES cells or that insertion of fluorescent protein genes into the ROSA26 locus elevates that propensity to undergo mitotic recombination. It is noteworthy that two other studies report values that are considerably lower, but still higher than those using Aprt as a reporter. ${ }^{34,35}$ A summary of 
several studies describing frequencies of mutation and LOH in ES cells has been described. 36

Impairment of mismatch repair in prokaryotes enhances recombination and the potential for lateral genomic transfer between species. ${ }^{37,38}$ The absence of effective mismatch repair produces a hyper-recombination phenotype in bacteria ${ }^{37,38}$ and possibly also in yeast. ${ }^{39}$ From an evolutionary perspective, it was not unreasonable to propose that mismatch repair proteins might control mitotic recombination, in part, in ES cells. Western blots of ES cell extracts show that mismatch repair proteins MSH2 and MSH6 are highly elevated in mouse ES cells compared with isogenic MEFs and that MLH1 and PMS2 are also elevated, but to a far lesser extent (Fig. 3). The role of mismatch repair in suppressing mitotic recombination has been examined using ES cells heterozygous at Aprt and transfected with an siRNA targeted to MSH2. Transfected cells were assessed for mutation frequency and frequency of LOH due to mitotic recombination, as was an ES cell line with an MSH2 missense mutant (G674A) which is devoid of mismatch repair activity. ${ }^{40}$ Figure 4 shows that when mismatch repair in mouse ES cells is impaired or absent, mutation frequency and mitotic recombination are elevated. One clone in which $\mathrm{MSH} 2$ has been reduced by about $60 \%$ showed about a 20-fold increase in mutation frequency and an almost proportional increase in LOH due to mitotic recombination. The G674A MSH2 mutant that is mismatch repair deficient showed an even greater elevation in mutation frequency and mitotic recombination, almost reaching the level observed in MEFs. In aggregate, the data indicate that one mechanism by which mouse ES cells preserve the integrity of their genomes is by suppressing mutation in general and mitotic recombination and consequent LOH in particular, the latter being mediated in part by proteins involved in mismatch repair.

\section{ES Cell Populations Retain Pristine Genomes by Eliminating Cells with Damaged DNA}

Mouse ES cells are hypersensitive to exogenous challenge and DNA damage, leading to apoptotic cell death. ${ }^{41-43}$ This may have advantages by ridding damaged cells from the selfrenewing stem cell population, thereby maintaining the genomes of the remaining cells pristine. Mouse ES cells lack a G1 checkpoint ${ }^{44,45}$ and the two major pathways that are function in somatic cells to activate the G1 checkpoint following introduction of DNA double strand breaks are compromised in ES cells. ${ }^{44}$ The pathways are presented as a simplified schematic in Figure 5. In brief, DNA lesions are probably sensed by the MRN complex (MRE11, RAD50, NBS1), resulting in phosphorylation and activation of ATM. When double strand DNA breaks are incurred, ATM initiates two pathways that result in the arrest of cells at a G1/S checkpoint. In one case, ATM phosphorylates p53 at serine 15, which contributes to p53 activation. P53 then induces transcription of the Cdk inhibitor p21, resulting in G1/S arrest. In the second case, ATM phosphorylates checkpoint kinase Chk2 on threonine 68 and promotes its activation. Activated Chk2, in turn, phosphorylates Cdc25A on serine 123 which contributes to its proteasome-mediated degradation. The Cdc25A bifunctional phosphatase dephosphorylates $\mathrm{Cdk} 2$ phosphothreonine 14 and phosphotyrosine 15 in the Cdk2/CyclinE and CyclinA complexes, promoting passage of cells from G1 into the S-phase. With diminished Cdc25A due to proteolytic degradation, or in its absence, Cdk2 is not dephosphorylated and cells are arrested in G1. Both of these pathways are compromised in mouse ES cells. ${ }^{44}$ In the first pathway, p53 is present but is predominantly

Adv Exp Med Biol. Author manuscript; available in PMC 2020 July 07. 
extranuclear in mouse ES cells, but not in somatic cells after irradiation. This is particularly well exemplified in Figure 6 where a colony of ES cells and a single MEF on the same coverslip show very different subcellular localization of p53 after being subjected to the same dose of radiation. Most of the detectable p53 is localized in the nucleus of the MEF whereas p53 is predominantly cytoplasmic in the cells of the ES cell colony. Consistent with this observation, p21 is not detectable in mouse ES cells, even after challenge by ionizing radiation. ${ }^{45}$

The second pathway appears to be regulated in part by the availability of Chk2. In mouse ES cells, Chk 2 colocalizes with $\gamma$-tubulin at centrosomes and is therefore likely unavailable to phosphorylate its substrates such as Cdc25A. In ES cells, Cdc25A is not degraded after exposure to ionizing radiation as it is in somatic cells and $\mathrm{Cdk} 2$ remains predominantly in its hypophosphorylated state, allowing unimpaired transit into S-phase. When ES cells are transfected with a plasmid encoding Chk2 so that Chk2 is expressed ectopically, Cdc25A is proteolyzed after irradiation and Cdk2 is phosphorylated, predictive of a G1 arrest. ${ }^{45}$ Consistent with this observation, ES cells expressing Chk2 ectopically and challenged with ionizing radiation display a G1 arrest (Fig. 7).

The operating hypothesis is that the absence of a G1 checkpoint allows damaged mouse ES cells to enter S-phase, where damaged DNA is replicated and the damage exacerbated with consequent cell death. Restoration of a G1 arrest in ES cells expressing Chk2 ectopically following exposure to ionizing radiation predicts that these cells should be protected from cell death. The prediction is borne out as described in Figure 8 where wildtype ES cells and ES cells expressing Chk2 ectopically were subjected to ionizing radiation and stained with propidium iodide as a vital stain and with Annexin $\mathrm{V}$ as an early marker of apoptosis. Populations of unchallenged mouse ES cells and ES cells expressing ectopic Chk2 are about $11 \%$ and $16 \%$ Annexin V positive, respectively, with very few dead (PI positive) cells. Following irradiation, about $40 \%$ of wildtype ES cells are Annexin $\mathrm{V}$ positive and a significant number are dead. In contrast, cells expressing ectopic Chk2 show no change in the number of apoptotic or dead cells (Fig. 8). The data again support the contention that the absence of a G1 checkpoint provides a mechanism to eliminate cells with damaged DNA from a population and thereby preserve the genomic integrity of the population as a whole.

\section{Mouse ES Cells Preferentially Utilize High-Fidelity Homology-Mediated Repair Rather Than Nonhomologous End-Joining to Repair Double Strand DNA Breaks}

When cells are exposed to certain therapeutics such as etoposide, a topoisomerase II inhibitor and radiomimetic, they sustain DNA damage including double strand DNA breaks. 46,47 The two major mechanisms by which this form of damage is repaired are the errorprone nonhomologous end joining (NHEJ) pathway and the high fidelity mechanism of homology-mediated repair. Although both mechanisms are operative in somatic cells, NHEJ is predominant and is active in all phases of the cell cycle. ${ }^{48}$ When considering the importance of genomic integrity for germ and ES cells, any error-prone mechanism of DNA repair would intuitively appear to be disadvantageous. To test the possibility that NHEJ is not the predominant pathway for repairing double strand DNA breaks in ES cells, the abundance of participating proteins in each of the pathways has been compared by Western

Adv Exp Med Biol. Author manuscript; available in PMC 2020 July 07. 
Blots between ES cells and isogenic MEFs. Notably, all of the proteins involved with homology-mediated repair are significantly elevated in extracts from ES cells compared with MEFs. In contrast, the levels of proteins involved with NHEJ are variable between ES cells and MEFs (Fig. 9). Of interest is that DNA Ligase IV, a rate-limiting enzyme in NHEJ is substantially lower in ES cells than in MEFs.

The abundance of proteins, while informative, is not necessarily indicative of function. The relative activities of both pathways have been examined in somatic cells and ES cells using a set of three reporter plasmids that collectively distinguish between homology-mediated repair and NHEJ. The plasmid for homology-mediated repair was pDR-GFP ${ }^{49}$ which has two tandem nonfunctional GFP genes separated by a puromycin resistance marker which allows for selection of stable transfectants. The $5^{\prime}$ gene is inactivated by insertion of an ISce1 restriction site that produces a frame-shift and a premature termination signal. The downstream GFP is truncated at both ends to render it inactive. A double strand break can be initiated at the I-Sce1 restriction site by the I-Sce1 enzyme. Repair of the GFP gene to functional status, mediated by recombination between the two nonfunctional fragments produces fluorescent cells that can be quantified. To assess NHEJ, two additional reporter plasmids have been used. The plasmid pEGFP-PEM1-AD2 ${ }^{50}$ also uses restoration of GFP fluorescence as a measure of activity, but the strategy allows fluorescence only after repair of a double strand break by NHEJ. In this reporter construct, two halves of the GFP gene are separated by an intron retaining its splice signals derived from the $P E M$ homeobox gene. Embedded within the PEM intron is an adenoviral AD2 gene that retains its own very strong splice acceptor and splice donor sites that perturb productive splicing and prevent restoration of GFP fluorescence. The AD2 gene is also flanked by paired Hind III sites and I-Sce1 sites in inverted orientation. When used in a transient transfection assay, the AD2 sequence is removed by digestion with I-Sce1 enzyme prior to transfection of cells. This also removes the its accompanying AD2 splice sites and leaves noncomplementary ends that can only be repaired by processing and NHEJ. When repaired, the PEM sequence is spliced out, the two GFP halves are brought into close proximity and the cells with repaired GFP are recognized and quantified by their fluorescence. The third reporter plasmid, designated pINV-CD $4,{ }^{51}$ is based on the expression of the surface marker CD4 following repair of a double strand break lesion by NHEJ. The parent plasmid contains a CMV promoter driving expression of the histocompatibility surface antigen $\mathrm{H}-2 \mathrm{Kd}$ followed by a CD8 cDNA in inverted orientation and a promoterless CD 4 cDNA. The $\mathrm{H}-2 \mathrm{Kd}$ and inverted CD 8 cDNAs are flanked by inverted I-Sce1 sequences that will delete the $\mathrm{H}-2 \mathrm{Kd}$ and $\mathrm{CD} 8$ sequences and yield noncomplementary ends when digested with I-Sce1 enzyme. When the ends are rejoined by NHEJ the CD4 surface marker is brought into close apposition to the CMV promoter and expressed. Cells in which the reporter plasmid has been repaired by NHEJ can be sorted and quantified by flow cytometry based on CD4 expression. The first and third reporter plasmids have been used in both stable and transient transfections.

As previously reported, somatic cells use predominantly NHEJ to repair double strand DNA breaks. Using the plasmids described above, this also appears to be true of MEFs, but not mouse ES cells, unless they are induced to differentiate. These data are summarized in Figure 10. Since MEFs and ES cells that had been induced to differentiate by culture with all-trans retinoic acid (ATRA) cannot easily be selected for stable reporter plasmid 
integrants, they were assayed for repair pathway utilization by transient transfection. Each cell population was electroporated with pDR-GFP after linearization by I-Sce1 digestion to assess homology-mediated repair. Repair by NHEJ was assessed by electroporation with pEGFP-PEM1-AD2 after I-Sce1 digestion. In both cases, efficiency of electroporation was determined by cotransfection with plasmid pDs-Red which emits red fluorescence. Efficacy of repair by each mechanism was based on the proportion of red fluorescent cells also had restored GFP-derived green fluorescence. The data confirmed that somatic cells utilize NHEJ predominantly for repair of double strand DNA breaks and demonstrate that ES cells preferentially utilize recombination-mediated repair for the same purpose. When ES cells are induced to differentiate, they switch their preferred pathway from recombinationmediated repair to NHEJ. Accompanying this switch is a reduction in the abundance of proteins, such as Rad51, that are involved in the recombination-mediated repair for the same purpose. When ES cells are induced to differentiate, they switch their preferred pathway from recombination-mediated repair to NHEJ. Accompanying this switch is a reduction in the abundance of proteins, such as Rad51, that are involved in the recombination-mediated process. There is also a significant increase in the level of DNA Ligase IV, which is particularly striking since all efforts to elevate the abundance of this protein in ES cells by overexpression or inhibition of its degradation were unsuccessful and led to rapid cell death. Thus, increased levels of DNA ligase IV that are not tolerated by ES cells appear to naturally accompany ES cells as they become differentiated and may help regulate NHEJ activity in somatic cells.

\section{CONCLUSION}

Historically, nuclei from somatic cells have been reported to have the capacity to support the development of a complete organism following transplantation into an enucleated egg, lending credence to the proposition that somatic cell nuclei retain sufficient integrity or "equivalence" to do so. The emergence IPS cell technology confirms the potential of somatic cell nuclei to undergo a dedifferentiation program and become pluripotent. The frequency of success with these techniques is usually very low, leading to concerns that the genomes of somatic nuclei, particularly from older individuals, are not equivalent to those of germ cells or ES cells and that the successes may come from a subset of cells that are not fully differentiated or that still maintain some stem-like properties. The low success frequencies may also be due, in part, to accumulated mutations in somatic cell nuclei that render many of the nuclei incapable of supporting complete conversion to pluripotency or to eroded telomeres that cannot always be restored. While these issues still remain to be resolved, they do call attention to the requirement for germ and ES cells to preserve the integrity of their genomes.

The present discussion has highlighted three mechanisms by which mouse ES cells or ES cell populations maintain genomic integrity. This does not imply that these mechanisms are unique to mouse ES cells or that these are the only mechanisms that can contribute to preservation of genomic stability. For example, human ES cells have more efficient mechanisms than somatic cells to repair types of DNA damage such as intrastrand crosslinks and 8-hydroxydeoxyguanosine lesions due to oxidative damage as well as double strand breaks. ${ }^{52}$ Mouse ES cells have more effective stress response mechanisms to render them 
more resistant to oxidative stress than fibroblasts. ${ }^{43}$ Human ES cells similarly have elevated expression levels of antioxidant genes SOD2 and $G P X 2$ and lower levels of reactive oxygen species (ROS) than differentiated cells, which may contribute to reduced levels of DNA damage due to endogenous sources. ${ }^{53}$ While less amenable to experimental manipulation and analysis than cells in culture, there is confirmatory evidence for protective strategies in vivo. Male germ cells in mice, for example, characteristically undergo extensive apoptosis $^{54,55}$ that occurs in massive waves. These occur during the time of primordial germ cell migration to the gonads and during the first round of spermatogenesis about 1' days postnatally, ${ }^{56}$ perhaps as a means to rid the germ cell population of damaged cells. It is also noteworthy that dividing spermatogonia are hypersensitive to radiation whereas the supporting Sertoli cells are far less so. ${ }^{57}$ Thus, Dawkins' concept ${ }^{58}$ reiterated by Weill and Radman "...that we indeed live as disposable somas, slaves of our germline genome..."23 is consistent with ever-increasing evidence that pluripotent cells, particularly cells of the germline, have developed multiple strategies for maintaining properties of self-renewal, pluripotency and essentially immortality.

\section{Supplementary Material}

Refer to Web version on PubMed Central for supplementary material.

\section{ACKNOWLEDGEMENTS}

The authors thank Drs. James Stringer, Alvaro Puga, El Mustapha Bahassi and Carolyn Price for their careful reading of the manuscript and their many helpful suggestions. This work was supported by grants from the NIEHS and the Center for Environmental Genetics at the University of Cincinnati.

\section{REFERENCES}

1. Ebert JD. Cell interactions: the roots of a century of research. Biol Bull 1985; 168:80-87.

2. Hämmerling $\mathbf{J}$ "Nucleo-cytoplasmic relationships in the development of Acetabularia". J Intern Rev Cytol 1953; 2:475-498.

3. Briggs R, King TJ. Transplantation of living nuclei from blastula cells into enucleated frogs' eggs. Proc Natl Acad Sci USA 1952; 38:455-463. [PubMed: 16589125]

4. Briggs R, King TJ. Nuclear transplantation studies on the early gastrula (Rana pipiens). Develop Biol 1960; 2:252-270. [PubMed: 13804423]

5. Gurdon JB. The developmental capacity of nuclei taken from intestinal epithelium cells of feeding tadpoles. J Embryol Exp Morphol 1962; 10:622-640. [PubMed: 13951335]

6. Evans M, Kaufman M. Establishment in culture of pluripotential cells from mouse embryos. Nature 1981; 292:154-156. [PubMed: 7242681]

7. Martin G Isolation of a pluripotent cell line from early mouse embryos cultured in medium conditioned by teratocarcinoma stem cells. Proc Natl Acad Sci USA 1981; 78:7634-7638. [PubMed: 6950406]

8. Doetschman T, Gregg RG, Maeda N et al. Targeted correction of a mutant HPRT gene in mouse embryonic stem cells. Nature 1987; 330:576-578. [PubMed: 3683574]

9. Thomas KR, Capecchi MR. Site-directed mutagenesis by gene targeting in mouse embryo-derived stem cells. Cell 1987; 51:503-512. [PubMed: 2822260]

10. Koller BH, Hagemann LJ, Doetschman T et al. Germ-line transmission of a planned alteration made in a hypoxanthine phosphoribosyltransferase gene by homologous recombination in embryonic stem cells. Proc Natl Acad Sci USA 1989; 86:8927-8931. [PubMed: 2573070] 
11. Zijlstra M, Li E, Sajjadi F et al. Germ-line transmission of a disrupted beta 2-microglobulin gene produced by homologous recombination in embryonic stem cells. Nature $1989 ; 342: 435-438$. [PubMed: 2685607]

12. Thomas KR, Capecchi MR. Targeted disruption of the murine int-1 protooncogene resulting in severe abnormalities in midbrain and cerebellar development. Nature 1990; 346:847-850. [PubMed: 2202907]

13. Wilmut I, Schnieke AE, McWhir J et al. "Viable offspring derived from fetal and adult mammalian cells". Nature 1997; 385:810-813. [PubMed: 9039911]

14. Campbell KHS, Fisher P, Chen WC et al. Somatic cell nuclear transfer: Past, present and future perspectives. Theriogenology 2007; 68(Suppl 1):S214-S231. [PubMed: 17610946]

15. Rideout III WM, Hochedlinger K, Kyba M et al. Correction of a genetic defect by nuclear transplantation and combined cell and gene therapy. Cell 2002; 109:17-27. [PubMed: 11955443]

16. Takahashi K, Yamanaka S. Induction of pluripotent stem cells from mouse embryonic and adult fibroblast cultures by defined factors. Cell 2006; 126:663-676. [PubMed: 16904174]

17. Takahashi K, Tanabe K, Ohnuki M et al. Induction of pluripotent stem cells from adult human fibroblasts by defined factors. Cell 2007; 131(5):861-872. [PubMed: 18035408]

18. Yu J, Vodyanik MA, Smuga-Otto K et al. Induced pluripotent stem cell lines derived from human somatic cells. Science 2007; 318:1917-1920. [PubMed: 18029452]

19. Szilard L On the nature of the aging process. Proc Natl Acad Sci USA 1959; 45:30-45. [PubMed: 16590351]

20. Kirkwood TB, Proctor CJ. Somatic mutations and ageing in silico. Mech Ageing Dev 2003; 124:85-92. [PubMed: 12618010]

21. Suh Y, Vijg J. Maintaining Genetic integrity in aging: a zero sum game. Antioxid Redox Signal 2006; 8:559-571. [PubMed: 16677100]

22. Stambrook PJ. Do embryonic stem cells protect their genomes? Mech Ageing Dev 2007; 329:313326.

23. Weil JC, Radman M. How good is our genome? Philos Trans R Soc London B 2004; 359:95-98. [PubMed: 15065661]

24. Shao C, Deng L, Henegariu O et al. Mitotic recombination produces the majority of recessive fibroblast variants in heterozygous mice. Proc Natl Acad Sci USA 1999; 96:9230-9235. [PubMed: 10430925]

24. Stambrook PJ, Shao C, Stockelman M et al. High frequency in vivo loss of heterozygosity is primarily a consequence of mitotic recombination. Cancer Res 1997; 157:1188-1193.

25. Stambrook PJ, Shao C, Stockelman M et al. Tischfield JA.APRT: a versatile in vivo resident reporter of local mutation and loss of heterozygosity. Environ Mol Mutagen 1996; 28:471-482. [PubMed: 8991080]

26. Kohler SW, Provost GS, Fieck A et al. Analysis of spontaneous and induced mutations in transgenic mice using a lambda ZAP/lacI shuttle vector. Environ Mol Mutagen 19991; 18:316321.

27. Van Sloun PP, Wijnhoven SW, Kool HJ et al. Determination of spontaneous loss of heterozygosity mutations in Aprt heterozygous mice. Nucleic Acids Res 1998; 26:4888-4894. [PubMed: 9776749]

28. Gossen J, de Leeuw WJ, Verwest A et al. High somatic mutation frequencies in a LacZ transgene integrated on the mouse X-chromosome, Mutat Res 1991; 250:423-429. [PubMed: 1944355]

29. Nohmi T, Katoh M, Suzuki H et al. A new transgenic mouse mutagenesis test system using spiand 6-thioguanine selections, Environ Mol Mutagen 1996; 28:465-470. [PubMed: 8991079]

30. Jakubczak JL, Merlino G, French JE et al. Analysis of genetic instability during mammary tumor progression using a novel selection-based assay for in vivo mutations in a bacteriophage lambda transgene target. Proc Natl Acad Sci USA 1996; 93:9073-9078. [PubMed: 8799156]

31. Cervantes RB, Stringer JR, Shao C et al. Embryonic stem cells and somatic cells differ in mutation frequency and type. Proc Natl Acad Sci USA 2002; 99:3586-3590. [PubMed: 11891338] 
32. Liu X, Wu H, Loring J et al. Trisomy eight in ES cells is a common potential problem in gene targeting and interferes with germ line transmission. Dev Dyn 1997; 209:85-91. [PubMed: 9142498]

33. Larson JS, Yin M, Fischer JM et al. Expression and loss of alleles in cultured mouse embryonic fibroblasts and stem cells carrying allelic fluorescent protein genes. BMC Mol Biol 2006; 7:36. [PubMed: 17042952]

34. Yusa K, Horie K, Condo G et al. Genome-wide phenotype analysis in ES cells by regulated disruption of Bloom's syndrome gene. Nature 2004; 429:896-899. [PubMed: 15215867]

35. Luo G, Santoro IM, McDaniel LD et al. Cancer predisposition caused by elevated mitotic recombination in Bloom mice. Nat Genet 2000; 26:424-429. [PubMed: 11101838]

36. Fischer JM, Stringer JR. Visualizing loss of heterozygosity in living mouse cells and tissues. Mutat Res 2008; 645:1-8. [PubMed: 18708075]

37. Vulic M, Lenski RE, Radman M. Mutation, recombination and incipient speciation of bacteria in the laboratory. Proc Natl Acad Sci USA 1999; 96:7348-7351. [PubMed: 10377417]

38. Matic I, Taddei F, Radman M. No genetic barriers between Salmonella enterica serovar typhimurium and Escherichia coli in SOS-induced mismatch repair-deficient cells. J Bacteriol 2000; 182:5922-5924. [PubMed: 11004198]

39. Manivasakam P, Rosenberg SM, Hastings PJ. Poorly repaired mismatches in heteroduplex DNA are hyperrecombinagenic in Saccharomyces cerevisiae. Genetics 1996; 142:407-416. [PubMed: 8852840]

40. Lin DP, Wang Y, Scherer SJ et al. An Msh2 point mutation uncouples DNA mismatch repair and apoptosis. Cancer Res 2004; 64:517-522. [PubMed: 14744764]

41. Van Sloun PPH, Jansen JG, Weeda G et al. The role of nucleotide excision repair in protecting embryonic stem cells from genotoxic effects of UV-induced DNA damage. Nucleic Acids Res 1999; 27:3276-3282. [PubMed: 10454634]

42. Roos WP, Christmann M, Fraser ST et al. Mouse embryonic stem cells are hypersensitive to apoptosis triggered by the DNA damage O6-methylguanine due to high E2F1 regulated mismatch repair. Oncogene 2007; 26:186-197. [PubMed: 16819506]

43. Saretzki G, Armstrong L, Leake A et al. Stress defense in murine embryonic stem cells is superior to that of various differentiated murine cells. Stem Cells 2004; 22:962-971. [PubMed: 15536187]

44. Aladjem MI, Spike BT, Rodewald LW et al. ES cells do not activate p53-dependent stress responses and undergo p53-independent apoptosis in response to DNA damage. Curr Biol 1998; 8:145-155. [PubMed: 9443911]

45. Hong Y, Stambrook PJ. Restoration of an absent G1 arrest and protection from apoptosis in embryonic stem cells after ionizing radiation. Proc Natl Acad Sci USA 2004; 101:14443-14448. [PubMed: 15452351]

46. Ferguson LR, Baguley BC. Topoisomerase II enzymes and mutagenicity. Environ Molec Mutagenesis 1994; 24:245-261.

47. Mclendon AK, Osheroff N. Topoisomerase II, genotoxicity and cancer. Mutat Res 2007; 623:8397. [PubMed: 17681352]

48. Mao Z, Bozzella M, Seluanov A et al. Comparison of nonhomologous end joining and homologous recombination in human cells. DNA Repair 2008; 7:1765-1771. [PubMed: 18675941]

49. Pierce AJ, Johnson RD, Thompson LH et al. XRCC3 promotes homology-directed repair of DNA damage in mammalian cells. Genes Dev 1999; 13:2633-2638. [PubMed: 10541549]

50. Seluanov A, Mittelman D, Pereira-Smith OM et al. DNA end joining becomes less efficient and more error-prone during cellular senescence. Proc Natl Acad Sci USA 2004; 101:7624-7629. [PubMed: 15123826]

51. Guirouilh-Barbat J, Huck S, Bertrand P et al. Impact of the KU80 Pathway on NHEJ-Induced Genome Rearrangements in Mammalian Cells. Molec Cell 2004; 14:611-623. [PubMed: 15175156]

52. Maynard S, Swistowska AM, Lee JW et al. Human embryonic stem cells have enhanced repair of multiple forms of DNA damage. Stem Cells 2008; 26:2266-2274. [PubMed: 18566332] 
53. Saretzki G, Walter T, Atkinson S et al. Downregulation of multiple stress defense mechanisms during differentiation of human embryonic stem cells. Stem Cells 2008; 26:455-464. [PubMed: 18055443]

54. Brinkworth MH, Weinbauer GF, Schlatt $S$ et al. Identification of male germ cells undergoing apoptosis in adult rats. Journal of Reproduction and Fertility 1995; 105:25-33. [PubMed: 7490711]

55. Richburg JH. The relevance of spontaneous- and chemically induced alterations in testicular germ cell apoptosis to toxicology. Toxicol Let 2000; 112-113:79-86.

56. Rodriguez I, Ody C, Araki K et al. An early and massive wave of germinal cell apoptosis is required for the development of functional spermatogenesis. EMBO J 1997; 16:2262-2270. [PubMed: 9171341]

57. Hasegawa M, Zhang Y, Niibe $\mathrm{H}$ et al. Resistance of differentiating spermatogonia to radiationinduced apoptosis and loss in p53-deficient mice. Radiat Res 1998; 149:263-270. [PubMed: 9496889]

58. Dawkins R The Selfish Gene Oxford University Press 1990. 


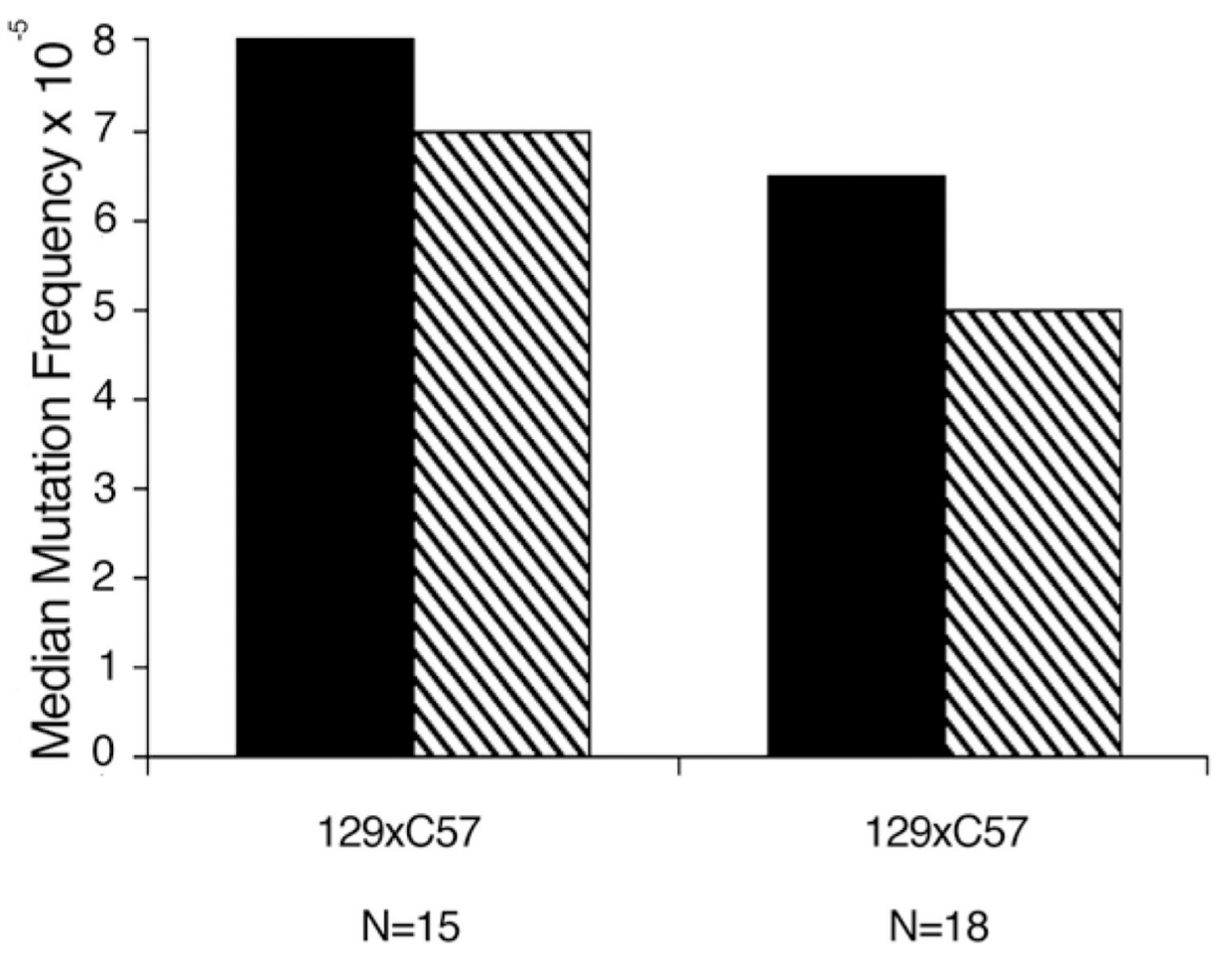

Figure 1.

Mutation frequencies and frequencies of mitotic recombination in skin fibroblasts and splenic T-cells in vivo. The solid bars represent total mutation frequency and diagonally striped bars represent the proportion of events due to mitotic recombination and consequent $\mathrm{LOH}$. 


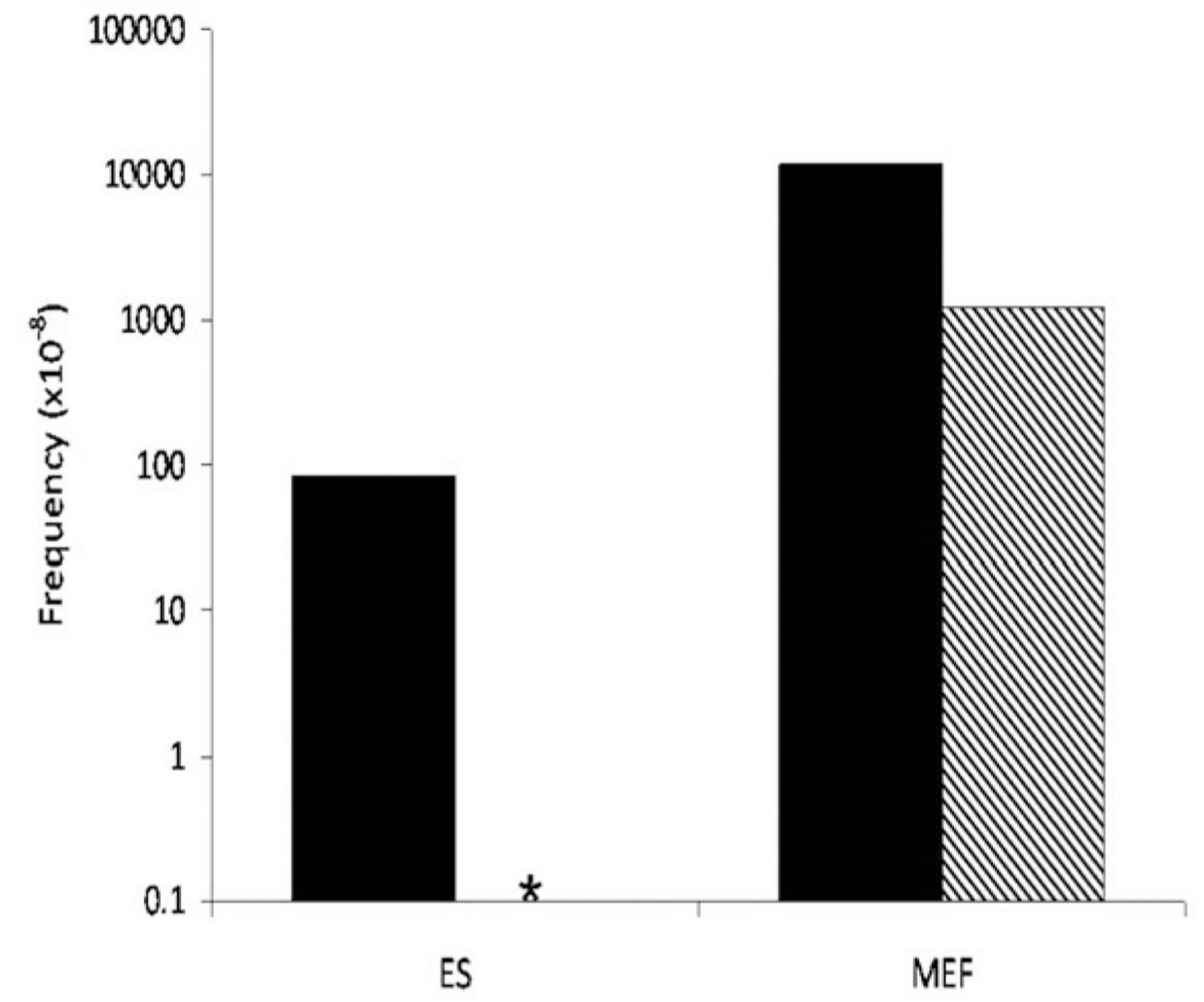

- Aprt $\mathbf{B} H$ prt

Figure 2.

Mutation frequency at Aprt (solid bars) and at Hprt (diagonally striped bars) in ES cells and MEFs. The asterisk indicates that no Hprt deficient colonies were detected in $10^{8}$ ES cells plated. The ordinate is on a logarithmic scale. 
MEF ES

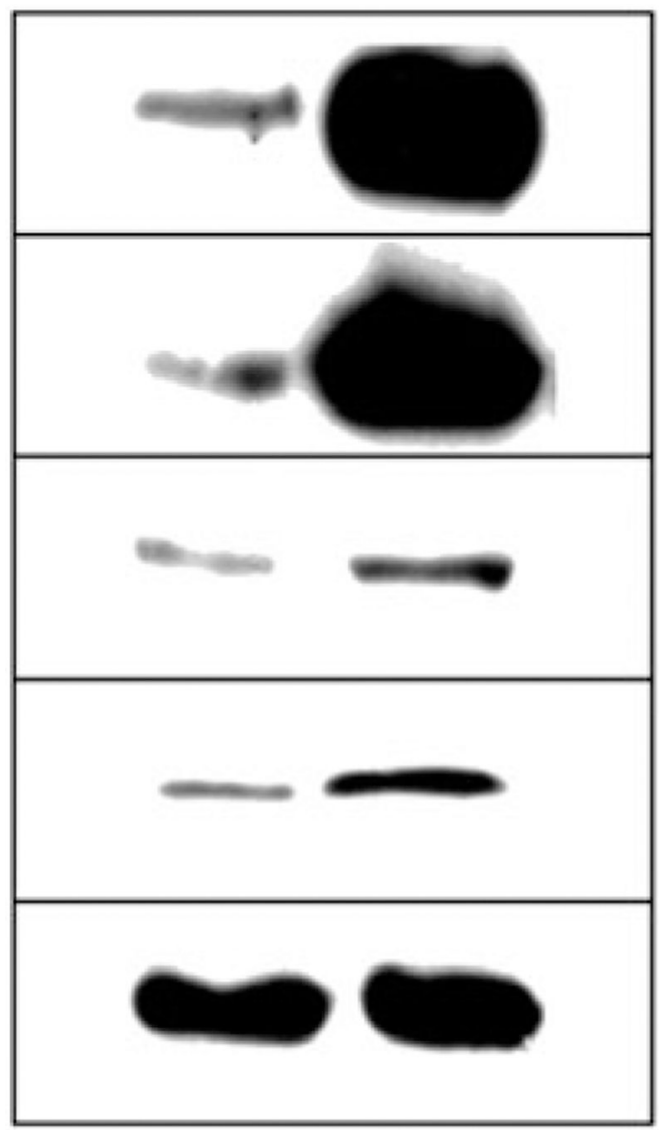

Msh2

Msh6

MLH1

PMS2

$\beta$-Actin

Figure 3.

Abundance of mismatch repair proteins in ES cells and MEFs. Cell lysates from ES cells and MEFs were subjected to Western blots following gel electrophoresis using antibodies to Msh2, Msh6, Mlh1 and Pms2. $\beta$-actin was used as a control. 


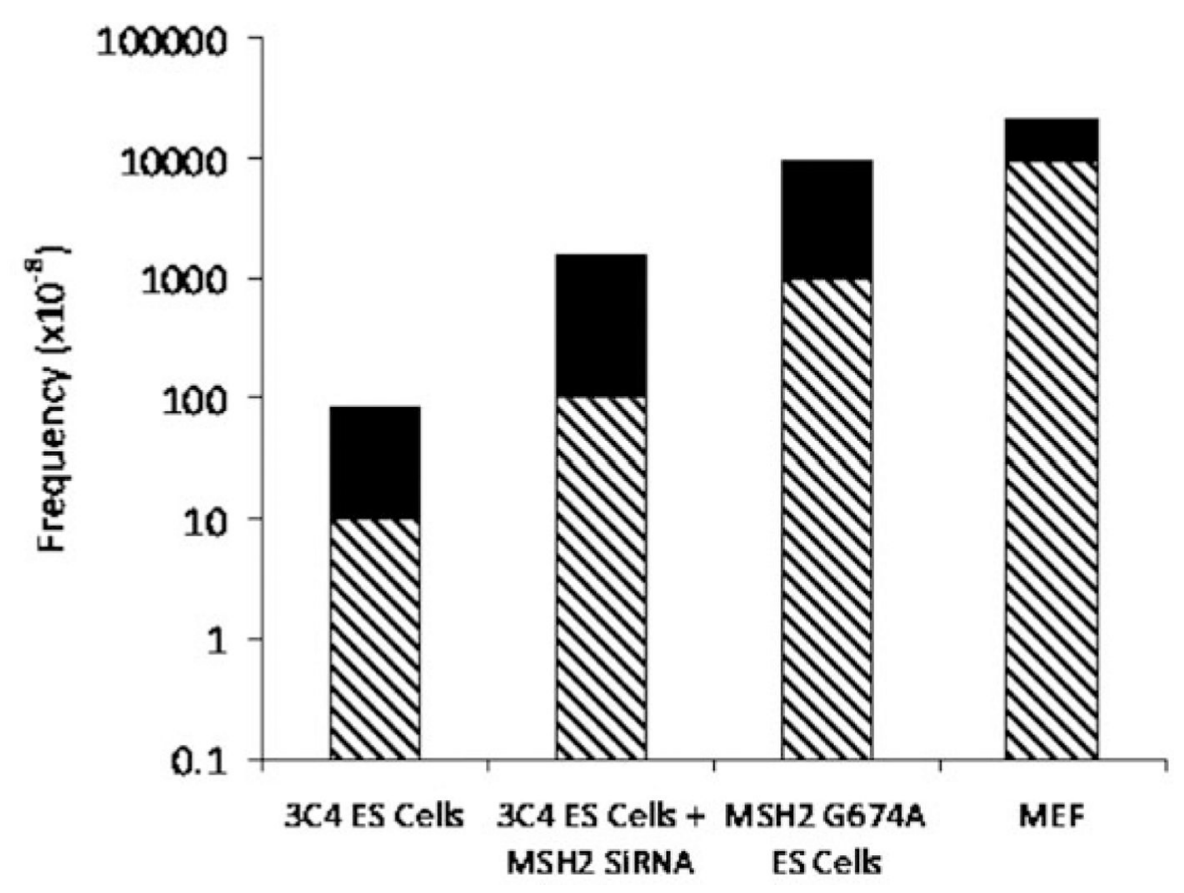

Figure 4.

Effect of reducing Msh2 on mutation frequency and mitotic recombination in ES cells. ES cells heterozygous at Aprt were transfected with siRNA to Msh2 and were then cultured in medium containing DAP. Colonies that arose were quantified and mutation frequencies normalized to colony formation in the absence of selection. The DNAs were then analyzed by PCR for mechanism by which the cells became Aprt deficient. 


\section{Double strand DNA break}

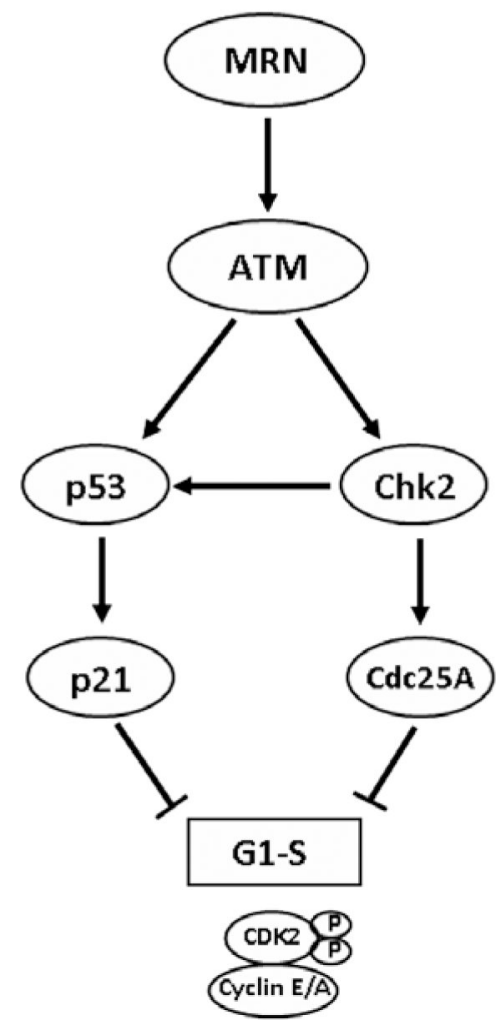

Figure 5.

Schematic showing the two major signaling pathways activated by double strand DNA breaks leading to $\mathrm{G} 1$ arrest. 
DNA

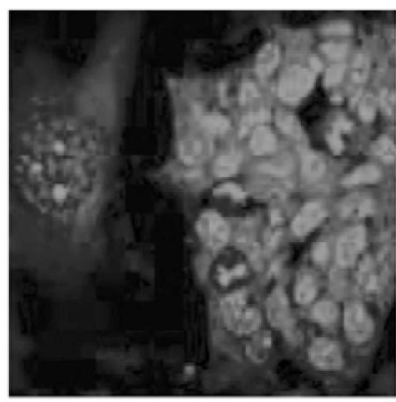

p53
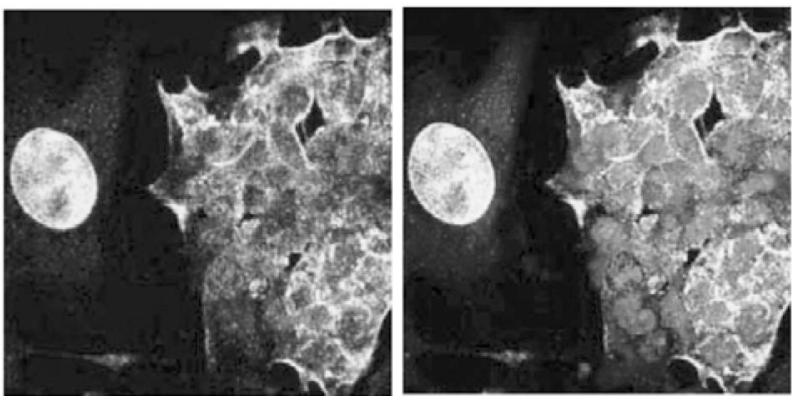

Figure 6.

Immunofluorescence of ES cells and a MEF on the same coverslip for p53 after $10 \mathrm{~Gy}$ Xirradiation. First panel shows DRAQ5 staining of nuclei. Note nucleus of MEF in the same field of focus as a colony of ES cells. Middle panel shows p53 staining after irradiation. The last panel is a merge of the first two images. 


\section{Unchallenged}
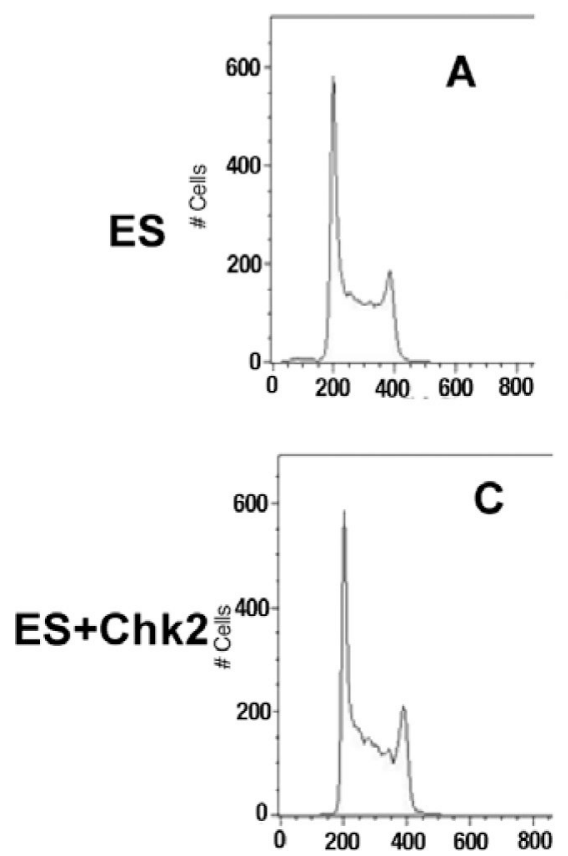

IR
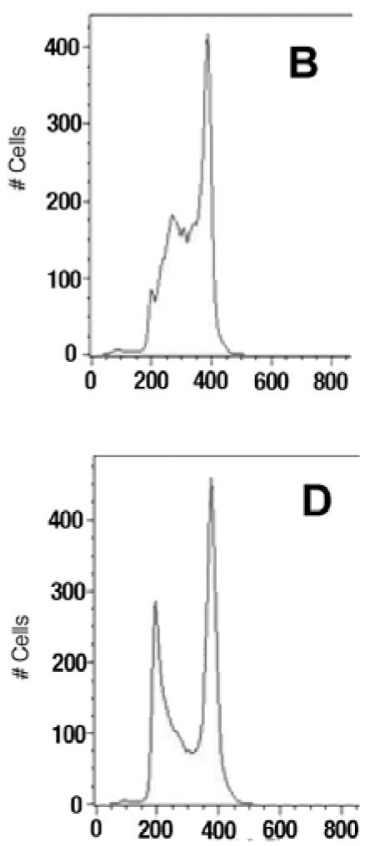

Figure 7.

Wildtype ES cells (panels A and B) and ES cells transfected with a plasmid encoding Chk2 were treated with ionizing radiation or left untreated, fixed, stained with propidium iodide and fractionated by flow cytometry to assess cell cycle distribution. In the absence of radiation (panels $\mathrm{A}$ and $\mathrm{C}$ ) the cellar distribution of both cell populations was similar. Following radiation, the wildtype cell population (panel B) showed a lack of cells in G1 with an accumulation of cells in S-phase and a clearly demarked G2/M cell cycle arrest.

Following radiation, ES cells expressing Chk2 ectopically retained the G2/M checkpoint and additionally showed that cells also were arrested in G1/S (panel D). 

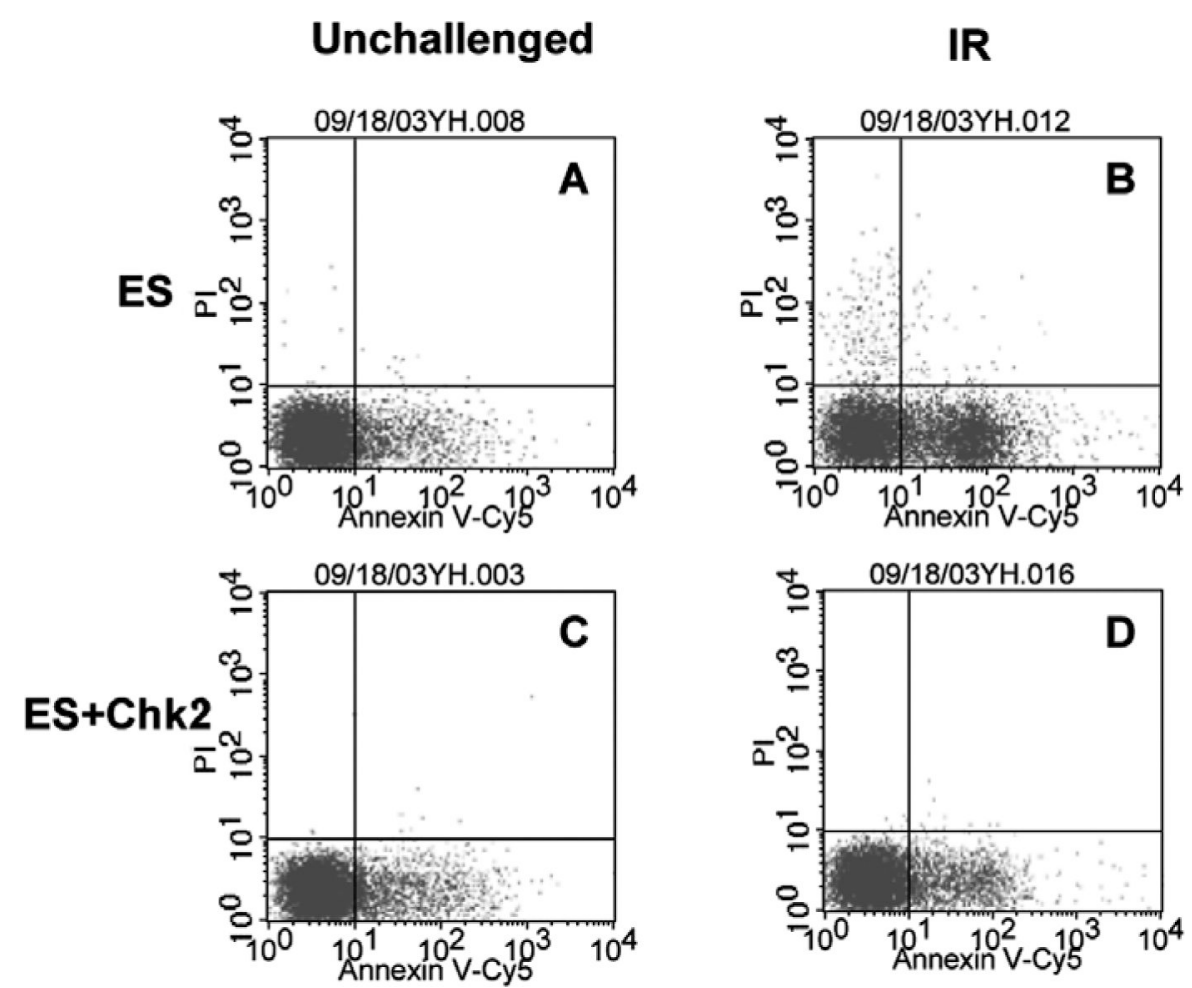

Figure 8.

Wildtype ES cells (panels A and B) and ES cells transfected with a plamid encoding Chk2 were treated with ionizing radiation (panels B and D) or left untreated (panels A and C), stained with Annexin $\mathrm{V}$ and propidium iodide and subjected to bivariate flow analysis. Cells above the horizontal line (PI positive) are dead and cells to the right of the vertical line are Annexin $\mathrm{V}$ positive (apoptotic). 


\section{Homology-Mediated Repair} MEF ES
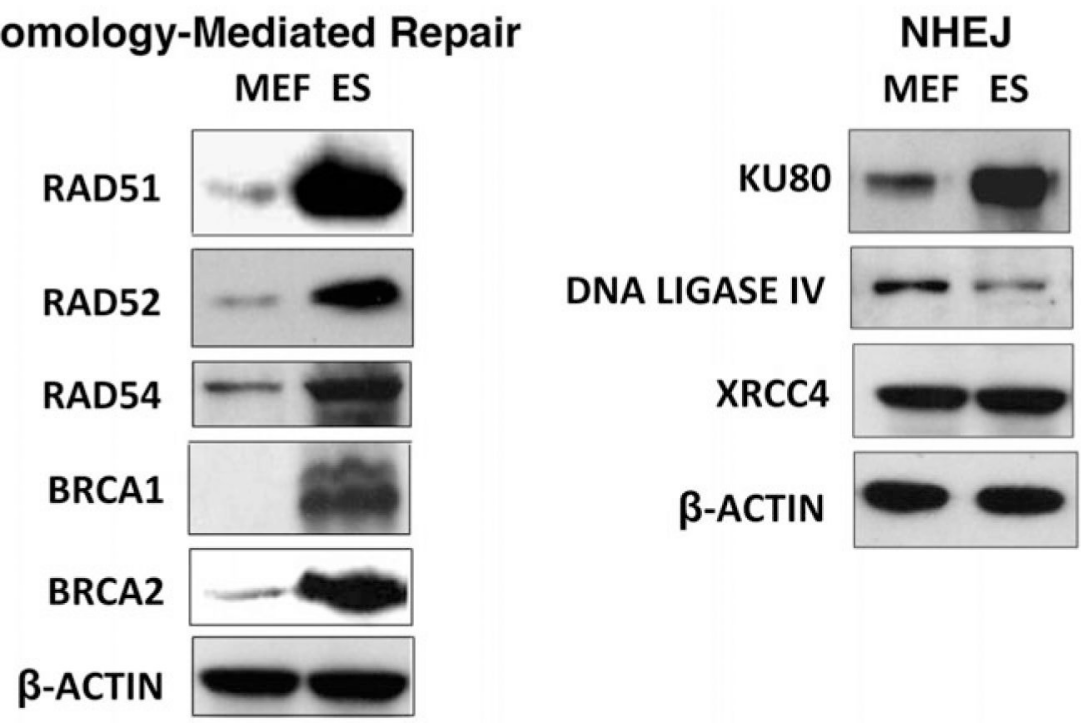

Figure 9.

Whole cell extracts prepared from ES cells and from MEFs were probed by Western blots for relative abundance of proteins involved in homology-mediated repair (left panel) and for NHEJ (right panel). 

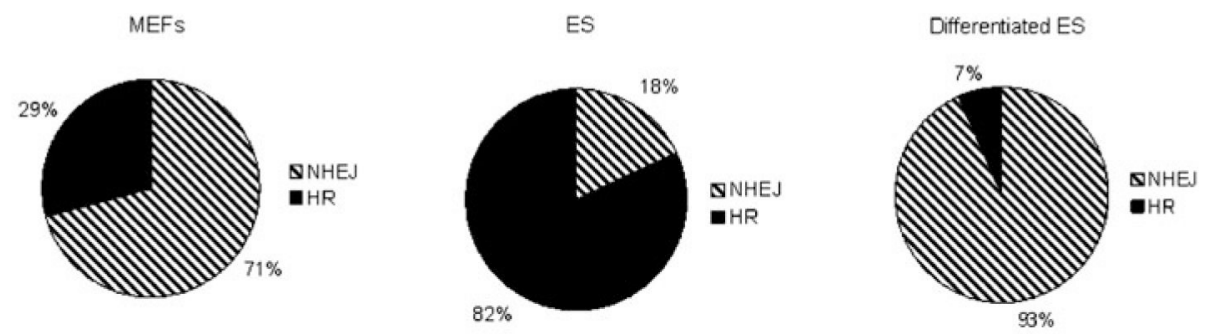

Figure 10.

Relative activities of double strand break repair by NHEJ (striped sectors) and by homologymediated repair (solid sectors) in MEFs, mouse ES cells and ES cells that had been induced to differentiate following culture in all-trans retinoic acid (ATRA). Cells of each population were electroporated with plasmid pDR-GFP after linearization with I-Sce1 to assess relative levels of recombination-mediated repair and with plasmid pEGFP-PEM1-AD2, also after ISce1 digestion to assess relative levels of NHEJ. In each case the assays were performed by transient transfection and quantification of cells that had acquired GFP fluorescence. 